\title{
Pathways to Development of Problem Gambling among Chinese Gamblers in Hong Kong: Validation of the Blaszczynski and Nower (2002) Model
}

\author{
Chi Chuen Chan ${ }^{1}$, Keis Ohtsuka ${ }^{2 *}$ \\ ${ }^{1}$ University of St. Joseph, Macau; ${ }^{2}$ School of Social Sciences and Psychology, \\ Victoria University, PO Box 14428, Melbourne, Vic 8001, Australia \\ *Corresponding author; email: keis.ohtsuka@vu.edu.au
}

\begin{abstract}
This study investigated the pathway to development of problem gambling in treatment-seeking gamblers attending a self-help group in Hong Kong. A total of 17 problem gamblers (15 men, 2 women; aged from 22 to 58 years) were interviewed and categorized according to the Blaszczynski and Nower (2002) pathways model. It was found that the majority of treatment-seeking gamblers were behaviorally conditioned gamblers. Gamblers of this type often reported an early involvement in gambling during childhood as part of family activities. They recalled an early win in their gambling history. Unlike gamblers with emotional problems in the Wood and Griffiths (2007) study, behaviorally conditioned gamblers exhibited little or no emotional problems before the onset of gambling problems. Further, their excessive gambling appears to be confounded with poor decision-making and a lack of effective coping skills. The findings of the current study also highlight the importance of family upbringing in the development of problem gambling.
\end{abstract}

Key words Chinese Gamblers · Gambling Pathways Model · Problem Gambling

\section{Introduction}

In recent years, there has been an increase in research interest in gambling in Hong Kong and Macau. The majority of studies have been prevalence studies (e.g., The University of Hong Kong, 2005; The Hong Kong Polytechnic University, 2002) or qualitative studies of the personality and social characteristics of gamblers (Cheng, 2006; Lam, 2004). In the prevalence study conducted by the University of Hong Kong (2005), $4.0 \%$ of the respondents were classified as problem gamblers and $1.8 \%$ as pathological gamblers. In the previous 12 months, $78 \%$ of the respondents 
had participated in gambling activities. More men than women had participated in the three most popular forms of gambling: 'Mark Six' (a type of lottery game), social gambling (mainly the game of mahjong played at home), and horse racing. In 2005, the participation rate in horse-race betting with the Hong Kong Jockey Club was $25.2 \%$ (cf, 30.4\% in 2001), while the participation rate in football betting in the previous 12 months was $6.3 \%$. The decrease in the participation rate in horse race betting, according to the authors, might also be the consequence of the legalization of football betting in 2003 because some gamblers placed bets on football pools instead of horses. The prevalence rate for probable problem gamblers was $3.1 \%$ (decrease from the $4 \%$ figure in 2001) and that of pathological gamblers was $2.2 \%$ (increase from $1.8 \%$ in 2001).

To date, there have been relatively few studies of the pathways to development of problem gambling in the Hong Kong population (Chan \& Ohtsuka, 2010; Wong, Leung, \& Lau, 2009). Wong and her associates, for example, analyzed the development of gambling behavior in eight men undergoing treatment for problem gambling in a treatment facility in Hong Kong, focusing on their narratives. In this study, the participants were asked to recount their childhood experiences, gambling initiation history, trajectories of problem gambling development and how their gambling behavior had impacted on their family members and themselves. One of the significant findings was the striking similarity in the early experience of problem gamblers in Hong Kong. All problem gamblers in the study reported that they grew up in a family that participated in gambling regularly and encouraged children to do so. Early exposure to gambling by way of family introduction and subsequent early success in gambling were prominent features in the development of their problem gambling behavior. Interestingly, the participants in Wong et al.'s study described the early gambling experience as 'normal'. Their involvement in gambling increased during their secondary school years and continued into young adulthood. It was not until they had suffered heavy gambling losses that they began to seek treatment for their gambling problems. The trajectories of the development of their gambling problem followed a linear progression model, from family gambling to social gambling in the adolescent years, to entrenched problem gambling in early adulthood. Wong et al.'s innovative study presents a rich phenomenological description of problem gamblers in Hong Kong through narrative analysis.

Chan (2011) and Chan and Ohtsuka (2010) investigated problem gamblers in Macau, known as 'Paichais', who spend most of their waking hours inside or in the vicinity of casinos. Chan employed qualitative research methods to investigate problem gamblers' views and analyzed their gambling careers from a perspective of the pathways model of pathological and problem gambling (Blaszczynski \& Nower, 2002). The researchers interviewed 15 active gamblers in the casinos of Macau and observed their gambling behavior. Results from these observations and subsequent interviews indicated that the pathways to development of pathological gambling followed a linear stage-based model. There were three stages seen in the formation of problem and pathological gambling. In the first stage, the participants often reported an early involvement in gambling during childhood. The majority of the problem gamblers reported coming from a family that encouraged gambling. 
They had a history of conduct disorder prior to the acquisition of their gambling habits. With increased involvement in gambling, these gamblers entered Stage 2 in the pathway to development of pathological gambling. In Stage 2, the participants often recalled more frequent and extensive involvement in gambling. They demonstrated symptoms of problem gambling, such as preoccupation with gambling, 'chasing' losses and incurring huge debts resulting from gambling losses. With increased immersion in gambling activities, these gamblers demonstrated significant detachment from other aspects of their lives outside casinos, as they spent most of their waking hours in the casinos. At this stage, the casinos had become their 'homes'. The behavior of the gamblers could now be described as being manipulative, cunning, socially irresponsible, impulsive and reckless, and the gamblers appeared to lack the ability to reflect on their actions. They were not motivated to seek treatment for their problems (as in the pre-contemplation stage, i.e., Prochaska \& DiClemente, 1986; Prochaska, DiClemente, \& Norcross, 1992; DiClemente et al., 1991).

Chan and Ohtsuka (2010) validated the Blaszczynski and Nower (2002) pathways model of problem gambling. In this model, all pathological gamblers can be classified into one of three groups: behaviorally controlled gamblers, emotionally vulnerable gamblers, or antisocial-impulsive gamblers. Chan and Ohtsuka (2010) found that most gamblers in their study were classified as antisocial-impulsive gamblers. Their findings differ from Wood and Griffiths' UK study (2007) in which the majority of pathological gamblers were classified as emotionally vulnerable gamblers. The UK gamblers mainly gambled to 'escape' from the problems of daily living. The differences between the two studies might be confounded by issues such as cultural differences, different types of gambling games, and the gambling history/trajectories of individual gamblers.

Cognitive factors such as misconceptions regarding odds and chances, subjective appraisal of probabilities and chance, and illusion of control further play a role in the maintenance of problem gambling behavior. For example, Chinese culture is considered to be considerably more superstitious in this regard than Western culture (Ohtsuka \& Chan, 2010; Papineau, 2005). Although the Chinese conceptualization of luck and winning may be unique to their culture, the underlying basic cognitive and psychological processes, such as attributions and motivations may reflect a universal framework (Ohtsuka \& Ohtsuka, 2010). Recent research by Oyserman and her colleagues reveals that culture fosters situated cognition, malleable rather than static interpretation, which is primed to influences cognitive task performance (Oyserman, in press; Oyserman et al, 2009).

The current study thus aimed to address gaps in existing research on culture and gambling behavior by recruiting participants in a gambling treatment center in Hong Kong. Firstly, the current study sought to understand the pathways to development of problem gambling among treatment-seeking gamblers in Hong Kong. Secondly, this research aimed to validate the typology of gamblers based on the Blaszczynski and Nower (2002) pathways model. To date, literature on the typology of Asian problem gamblers is scarce and this research thus aimed to address this existing gap in knowledge. 


\section{Method \\ Participants}

A total of 17 participants (15 men, 2 women; aged from 22 to 58 years; $M$ age = $40.35, S D=10.65$ years) were recruited from a self-help group for problem gamblers in Hong Kong. Their involvement in the self-help group ranged from 1 month to 7 years ( $M$ participation duration $=24$ months). The majority of the participants (13) worked in semi-skilled jobs. Only two classified themselves as 'professionals' (a secondary mathematics teacher and an investment banker). Men outnumbered women at the meetings. Most of the participants had completed at least secondary school education. Two participants had a college education. Another participant had completed a postgraduate degree. The majority of the participants did not disclose their monthly salaries. The authors speculate that the reluctance to report income levels might reflect Chinese culture, where levels of income are seen as indicators of success in one's career and are directly related to self-esteem. In total, 18 members of the self-help group were invited to participate in the study. One declined and 17 members agreed to participate (participation acceptance rate of 95\%). Pseudonyms are used in this article to protect participants' identities.

\section{Measures}

Observation data and interview data were collected over 6 months from November 2009 to May 2010. Self-help group meetings were held once every week on a Wednesday night in a church. Usually, the meetings began with a short introduction of the attending members and a welcome to new members. The self-help group members were encouraged to disclose their history of gambling behavior and their attempts at abstinence. The first author attended group meetings from November 2009 to May 2010 three times. On these occasions, during weekly meetings, the researcher played the role of a meeting moderator under the supervision of the group coordinator. The participants in these meetings, who had given consent to participate in the research project, were aware that their views and opinions may be included in the research report without identifiable information (to protect their identity). The one-to-one interviews for participants were carried out separately from the self-help group session meetings. A copy of the interview guide is available from the corresponding author on request.

\section{Procedure}

The first author of this article served as a voluntary counselor for a self-help group for problem gamblers in Hong Kong from 2009 to 2010. This self-help group ran the group sessions based on a Gamblers Anonymous paradigm where the participants met weekly in group meetings held in a church. Group meetings placed the emphasis on sharing, self-revelation and self-learning of abstinence from gambling. The weekly meetings were conducted in Cantonese, the official language of the Hong Kong Special Administrative Region, China. Referral to the self-help group came from two sources: self-referrals and referrals by family members. The meetings reflected a Christian perspective, with prayers and references to biblical readings made during the course of the gatherings.

The first author obtained consent and permission from the chairperson of the 
self-help group. The research was conducted in accordance with the code of conduct for staff members at the University of St. Joseph in Macau. One month prior to the start of the current investigation, the first author contacted the self-help group facilitator, who subsequently introduced the first author to the members of the group. During the course of the data collection, the participants were interviewed by the first author, a clinical psychologist practicing in Hong Kong. The semistructured interview schedule included questions on the participant's family and career background, history and trajectory of gambling, as well as the causes and triggers of gambling and the impact of gambling on their lives. As each participant differed in their life experience, the researcher's interview reflected this in greater emphasis on particular themes and concepts as appropriate to each participant. For example, if a participant came from a single parent family, the interview would place more emphasis on this theme and would explore how this factor had contributed to the development of the participant's gambling habit. After each interview, the researcher transcribed the participants' narrative stories. Then, thematic analysis was undertaken using the transcribed notes.

The data analysis followed Hycner's (1999) explication process, specifically: (a) bracketing and phenomenological reduction; (b) delineating units of meaning in the participant's interview responses and examining how these would contribute to the holistic meaning of the participant's worldview; (c) clustering of units of meaning to form themes; (d) summarizing and validating each interview in context; and (e) extracting and developing general and unique themes as a summary of interview data.

Operational definitions for gambling behaviors were used in order to complete a functional assessment of the participants. For example, impulsivity was given the operational definition: 'the inability to resist the urge to gamble, always wagering despite repeated losses and failure to stop gambling and inability to leave the casino even after gambling funds are exhausted'. Chasing was defined as: 'returning to the casino on the next day to recover one's loss'. Conduct disorder was defined as: 'the type of behavior that runs contrary to social rules, norms and expectations.' An example of this is stealing from school and friends. For instance, conduct disorder in high school was defined by evaluating the extent of the disciplinary problems reported by the participants. These disciplinary problems included truancy, risky sexual behavior and substance abuse.

Classification of the pathway to development of gambling problems was achieved through review of each person's behavior during their high school years. Further, the assessment of the participant's involvement in gambling was assessed according to criteria from the Diagnostic and Statistical Manual $4^{\text {th }}$ ed., text-revision (DSM-IV-TR) (American Psychiatric Association, 2000). The criteria for diagnosis of pathological gambling are: preoccupation with gambling, tolerance, withdrawal, loss of control, gambling to escape, chasing, lying, illegal acts and relationships being at risk due to gambling. Participants demonstrating five or more criteria were diagnosed as pathological gamblers.

\section{Results}

Results of the clinical interviews formed the basis of the present findings. Of the 17 participants, 16 were classified as pathological gamblers according to the DSM-IV- 
TR (2000) criteria. Only one participant, Bill (male, 53 years old), was classified as a social gambler. He attended the self-help meetings regularly with his son, James (male, 31 years old), who was a pathological gambler. Bill participated in the selfhelp group meetings mainly to accompany and support his son.

Results of the thematic analysis from the transcribed interviews were interpreted in reference to the Blaszczynski and Nower's (2002) pathways model of pathological gambling. On the basis of the interview and group discussion, the first author, a clinical psychologist specializing in problem gambling issues, classified participants' behavior using the criteria in the DSM-IV-TR (American Psychiatric Association, 2000). Approximately half of the participants $(n=9$ or $52.9 \%)$ were classified as behaviorally conditioned gamblers, four (23.5\%) as antisocial-impulsive gamblers and three participants $(17.6 \%)$ as emotionally vulnerable gamblers. The development of their gambling behavior and personality characteristics are detailed in the following section.

\section{Early years}

The majority of participants reported involvement in gambling during childhood, with gambling a part of family games and pastimes. For example, Ron (male, 40 years old) reported that he started gambling at 10 years of age. Dick (male, 46 years old) said that he started gambling at 7 years of age. In their recollections, gambling was taken as a normal and fun activity during their childhood - it was a part of their shared memories with family members, relatives, and friends. The most common gambling game played at home was mahjong. According to the participants, gambling was a normal part of their early lives. Such 'normalcy' has also been reported by Wong et al. (2009) in a study in which they analyzed the narratives of problem gamblers in treatment. In the interviews with participants in the current study, almost all used the words "to play" to describe their early family introduction to gambling (rather than "to gamble"). Some of their favorite gambling games when they reached early adulthood were dice and card games. The notion of play was evidenced in their description of childhood games. Ron (40 years old) described himself as a thrill seeker who liked to gamble on almost anything. Beginning at 10 years old of age, he had started gambling on mahjong and later was heavily involved in commercial gambling, such as horse-racing, casino gambling and soccer betting. During the interviews, he boasted of his knowledge and expertise in gambling games. He believed that through hard work and diligence, he could improve his chance of winning gambling games. Throughout his life since childhood, gambling was his major interest because he believed that he could make a fortune from gambling.

As these gamblers progressed on their gambling pathways, they usually recalled experiencing early big wins as part of the development of their gambling problem. For example, John (male, 28 years old) had a big win in the casinos in Macau. Coming from an impoverished background, a win of HKD 100,000 (approximately AUD 12,066) was a fortune to him. John confided to the first author that he spent most of his winnings on prostitutes. After he spent all the gambling winnings, he resumed his former job as a sanitation worker.

Most of the gamblers relished the extravagance and 'bravado' of buying 
their friends expensive gifts and lavishing them with expensive dinners from their gambling winnings. Gambling winnings were a means to boost their egos. It has been suggested that Chinese gamblers have a special liking for having admirers or being followers of expert gamblers during their gambling sessions (Chan \& Ohtsuka, 2010). Mass media, such as movies, often portray a social pecking order among gamblers based on their skill, expertise and gambling prowess (Chan \& Ohtsuka, 2011; Ohtsuka \& Chan, 2009). Chinese gamblers, especially male gamblers, enjoy being "the big boss" or "gambling master." Doug (male, 56 years old) recalled that he used to bring a number of his followers on a gambling trip to Macau. If he won a large amount of money, he distributed some of his earnings among his followers to impress them. This type of bravado and ego boosting is undoubtedly a reinforcer of gambling behavior, especially for gamblers from a low socioeconomic status who otherwise are unable to attain such a desirable status and the admiration of such followers.

The majority of participants shared similar characteristics at childhood. Out of this similar family environment, however, three types of pathological gamblers emerged at a later stage in life. The first of these types is the behaviorally conditioned gambler ( $n=9$ or $52.9 \%$ ), as defined in Blaszczynski and Nower's (2002) pathways model of problem and pathological gambling. Behaviorally conditioned gamblers usually do not have any emotional or psychological problems prior to their acquisition of gambling behavior. For these gamblers, gambling is a fun pastime shared with friends and family members. Doug (male, 56 years old), for example, described his life as a normal, unremarkable one despite the repeated gambling losses he suffered at the casino tables. For these gamblers, gambling is considered to be their only pastime. For instance, M.C. (male, 30 years old) said that he had no other hobby other than gambling. M.C. appeared to have underlying psychological issues. Having completed only primary school education, he had not achieved anything of significance in terms of career development. Further, M.C. did not have contact with his family or socialize with close friends. Gambling appeared to be an 'attachment figure' around which he constructed meaning for his life.

An interesting finding among the behaviorally conditioned gamblers in this study was that gambling was their sole pastime or hobby. Some gamblers even went further to assign to gambling a special meaning through which they achieved a higher self-esteem vis-a-vis their peers. For example, they would proudly recall instances where they bought dinners or expensive gifts for friends with gambling winnings they had made in the casinos. They had lived a normal life prior to the acquisition of their gambling habits. Their emotional problems were therefore more likely to be a consequence of their gambling rather than the cause of their gambling addiction. More often than not, their excessive gambling seemed to be a consequence of poor decision making and judgment and a lack of effective coping skills.

The behaviorally conditioned gamblers in the current study were amenable to therapeutic intervention because they had significant insight to identify the root causes of their gambling problems. They were able to reflect and empathize with other group members. One such example was Tommy (male, 52 years old) who started gambling at the age of 15 . Subsequently married and with a teenage daughter, 
Tommy had been gambling for over 30 years. He would have been classified as a pathological gambler prior to joining the self-help group. In his own description, he had significant difficulty in controlling his impulses to gamble. However, unlike antisocial-impulsive gamblers in the current study (to be discussed in the next section), Tommy was able to empathize with the feelings of his family. Regular attendance at the self-help group contributed to his recovery from pathological gambling. Tommy had been able to abstain from gambling in the two years prior to the current research interview.

The second type of pathological gamblers identified in the study $(n=4)$ were classified as antisocial-impulsive gamblers according to the Blaszczynski and Nower (2002) pathways model. These gamblers share a similar development pathway with the first group of gamblers - all four gamblers reported coming from a family that encouraged gambling or 'hanging out' with gambling friends during their early years. Gambling was a part of their childhood. One significant factor that distinguished this group from the behaviorally conditioned gamblers was their lack of attachment to family during their early formative years. One example is Sam (male, 21 years old), a new immigrant from Mainland China. Sam spent his childhood as an abandoned child in his village. He barely finished school and had not achieved anything of note in terms of career development. He described himself as an undisciplined and uneducated child during his childhood years. He tended to use people for his own benefits. He reported resorting to lying in order to obtain funds for his gambling. The researcher also interviewed his wife. She described Sam as a pathological liar who manipulated others for his own gain. Sam joined the self-help group to please his family but stopped attending meetings a few months later. During interviews, Sam was never able to admit his own problems. As a group, these gamblers demonstrated many of the antisocial-impulsive behavior characteristics of Type 3 gamblers in the Blaszczynski and Nower (2002) model. These behaviors include impulsiveness, recklessness and difficulty in delaying gratification with respect to needs and wants. These gamblers have been described as being indifferent to social norms, unreliable, and irresponsible and likely to have difficulties in following a law-abiding career (Blaszczynski \& McConaghy, 1994; Blaszczynski \& Steel, 1998).

The third group of gamblers $(n=3)$ could be described as emotionally vulnerable gamblers. These gamblers had significant personal/emotional problems prior to their acquisition of gambling behavior. One such example is John (male, 28 years old). With divorced parents, John described himself as having been an unhappy child. His mother was a gambler, who left him with his father. John was an underachiever at school who 'dropped out' of high school. At the age of 28, he was working as an apprentice in the construction industry. By his own admission, he seldom put his attention to planning anything significant in his life. He stated that he often felt an 'emptiness' inside and that he would gamble to numb such feelings. John's personality profile fits with a diagnosis of borderline personality disorder. People of this personality type often describe themselves as emotionally vulnerable, with instability in relationships, mood, and self-image. They often report chronic feelings of emptiness; uncertain and/or changeable self-image (i.e., values, loyalties, identity) and extreme mood swings. For individuals with 
borderline personality disorder, gambling might be a behavior which serves to fill the emotional void. They are also vulnerable to being influenced by 'big spenders' or expert gamblers and to attempt to follow in their footsteps. The current findings regarding this subtype of problem gamblers are consistent with those of Wood and Griffiths (2007) who found that dissociation gambling to 'escape' daily worries was the major motivation for gambling among emotionally vulnerable gamblers.

\section{Discussion}

The present study has provided evidence in support of the Blaszczynski and Nower's (2002) pathways model of problem and pathological gambling. The current findings clearly demonstrate that there are three distinct developmental pathways in pathological gambling. The current study also supports the claims of Wong et al. (2009) about family providing the introduction to gambling for problem gamblers in Hong Kong. The majority of the gamblers in the study reported that they came from a family environment that encouraged them to gamble. This finding echoes the suggestion by Papineau (2005) that Chinese children are more susceptible to gambling because gambling is considered to be a family activity in Chinese culture. In Papineau's paper on the Chinese immigrant community in Montreal, she argued that the Chinese family fosters gambling through parental modeling and tutelage in childhood. Further, the Chinese concept of luck and winning, the ebb and flow to be decoded by a celestial calendar, further encourages continuous involvement in gambling in her view (Papineau, 2005). Although it could be possible that such a view is culturally prevalent interpretation (Oyserman, in press; Oyserman et al., 2009), we argue that gamblers' ability to adopt an alternative perspective is rather limited if not impossible. Research evidence on gambling-related cognitive distortions, such as 'gambler's fallacy'(e.g., Delfabbro, 2004), clearly demonstrates tenacious persistence of such misconceptions.

Secondly, the present study highlighted that personality disorder is often associated with problem gambling. Among the participants, four could be classified as exhibiting antisocial personality disorder and one could be diagnosed as having a borderline personality disorder. Blaszczynski and his associates (Blaszczynski \& McConaghy, 1994; Blaszczynski \& Steel, 1998) argue that personality disorders are often a co-morbid condition of pathological gambling. In the current study, it was noted that personality disorders preceded problem gambling for some treatmentseeking gamblers. The case with the borderline personality disorder described previously is a good illustration, with this individual gambling to cope with his personal problems. Thus, problem gambling for this group fundamentally stems from issues related to their personality disorders.

Thirdly, the present study supports the claim that the presence of impulsiveness differentiates the emotionally vulnerable gamblers and the antisocial gamblers (Haw, 2009). The antisocial-impulsive gamblers in the study demonstrated no signs of depression. By contrast, depressive moods were very common among the emotionally vulnerable gamblers. Two distinctively different personality profiles among problem gamblers further support the notion that multiple pathways lead to the formation of problem and pathological gambling (Blaszczynski \& Nower, 2002). 
Lastly, this study demonstrated that self-help groups are a viable means to help gamblers. The study demonstrates that the crucial aspects of this help are sharing and mutual support (Taber \& McCormick, 1987; Toneatto \& Millar, 2004; Wong et al., 2009).

The present study sought to understand the aetiology of pathological gambling in a self-help group. The results from the small sample size of 17 participants might not be representative of gamblers as a whole. Self-help groups are known to have a high rate of attrition or 'dropouts' (Wong, 2010). The fact that the behaviorally conditioned gamblers comprise the majority of treatment-seeking problem gamblers in this study might indicate a sampling bias. It is possible that antisocialimpulsive gamblers may not come to treatment meetings. In fact, the majority of antisocial-impulsive gamblers may not be aware of the need for treatment - they may be permanently in the pre-contemplation stage according to Prochaska et al.'s (1992) stage of change model. Further, the current study was mainly based on the recollections of problem gamblers in treatment. As such, the primary data were obtained from interview sessions of gamblers' recollection of their gambling introduction, history, development and understanding of the underlying issues leading to their gambling problem. The reliability of these reports can be questioned, especially for participants who have been abstinent for many years. The length of lapsed time may result in inadvertent but incorrect recall of information.

In addition, problem gambling carries substantial negative stigma even for treatment-seeking gamblers who have taken decisive steps to address the cause of their gambling. Due to the sensitive nature of the subject matter, participants may opt not to discuss embarrassing or socially undesirable aspects by self-censoring their recollections. Similarly, treatment-seeking problem gamblers may exaggerate the success of the recovery process or their record of abstinence while hiding instances of gambling relapses.

Lastly, the self-help paradigm which emphasizes gambling abstinence in a church setting reflects the strong influence of the alcoholics anonymous approach developed in western culture. Its effectiveness for Chinese problem gamblers whose culture is substantially different from their western counterparts could be a further topic of investigation. These limitations withstanding, the current research highlighted common features and differences in personality profiles found in the group of treatment-seeking problem gamblers in Hong Kong. The findings of the current study provide support for the Blaszczynski and Nower's (2002) pathways model of problem and pathological gambling.

Recommendations for future studies of Chinese gamblers include recruitment of participants from active current gamblers exhibiting no signs of problem behavior to gain a better understanding of the pathways development of problem and pathological gambling. Such research is important to further shed light on the aetiology of problem gambling in Hong Kong.

\section{Acknowledgment}

The authors would like to thank the chairperson of the self-help group and the group members who participated in the study. 


\section{References}

American Psychiatric Association. (2000). Diagnostic and statistical manual of mental disorders (4th ed, text revised). Washington, DC: American Psychiatric Association.

Blaszczynski, A., \& McConaghy, N. (1994). Antisocial personality disorder and pathological gambling. Journal of Gambling Studies, 10, 129-146.

Blaszczynski, A., \& Nower, L. (2002). A pathways model of problem and pathological gambling. Addiction, 97, 487-499.

Blaszczynski, A., \& Steel, Z. (1998). Personality disorders among pathological gamblers. Journal of Gambling Studies, 41, 51-71.

Chan, C. C. (2011). The clinical and social construction of pathological gamblers in Macau (Unpublished doctoral dissertation). Victoria University, Melbourne, Australia.

Chan, C. C., \& Ohtsuka, K. (2010). The clinical and social construction of Chinese pathological gamblers in Macau. Proceedings of the International Conference on Gaming Industry and Public Welfare 2010 November 2010, Beijing, China (pp. 254-259).

Chan, C. C., \& Ohtsuka, K. (2011). All for the winner: An analysis of the characterization of male gamblers in Hong Kong movies with gambling theme. International Journal of Mental Health and Addiction, 9, 208-218. doi:10.1007/s11469-010-9274-5

Cheng, W. K. (2006). From pathological gambling to help seeking: Cases offemale pathological gamblers in Hong Kong (Unpublished master's thesis). The University of Hong Kong, Hong Kong.

Delfabbro, P. (2004). The stubborn logic of regular gamblers: Obstacles and dilemmas in cognitive gambling research. Journal of Gambling Studies, 20, 1-21. doi: 10.1023/B:JOGS.0000016701.17146.d0

DiClemente, C. C., Prochaska, J. O., Fairhurst, S. K., Velicer, W. F., Velasquez, M. M., \& Rossi, J. S. (1991). The process of smoking cessation: An analysis of precontemplation, contemplation, and preparation stages of change. Journal of Consulting and Clinical Psychology, 59, 295-304.

Haw, J. (2009). Impulsivity partially mediates the relationship between depression and problem gambling, Gambling Research, 21, 12-21.

Hycner, R. H. (1999). Some guidelines for the phenomenological analysis of interview data. In A. Bryman \& R. G. Burgess (Eds.), Qualitative research (pp. 143-264). London: Sage.

Lam, H. P. (2004). Becoming pathological casino gamblers in Hong Kong: Do big winning experiences matter (Unpublished master's thesis)? The University of Hong Kong, Hong Kong.

Ohtsuka, K., \& Chan, C. C. (2009). Desperate housewives: An analysis of the characterisations of female gamblers portrayed in gambling movies in Hong Kong. International Journal of Mental Health and Addiction, 7, 229-238. doi:10.1007/s11469-008-9180-2

Ohtsuka, K., \& Chan, C. C. (2010). Donning red underwear to play mahjong: superstitious beliefs and problem gambling among mahjong players in Macau. Gambling Research, 22, $18-33$.

Ohtsuka, K., \& Ohtsuka, T. (2010). Vietnamese Australian gamblers' views on luck and winning: Universal versus culture-specific schemas. The Asian Journal of Gambling Issues and Public Health, 1, 34-46.

Oyserman, D. (in press). Culture as situated cognition: Cultural mindsets, cultural fluency and meaning-making. European Review of Social Psychology.

Oyserman, D., Sorensen, N., Reber, R., \& Chen, S. X. (2009). Connecting and separating mindsets: Culture as situated cognition. Journal of Personality and Social Psychology, 97, 217-235.

Papineau, E. (2005). Pathological gambling in Montreal's Chinese community: an anthropological perspective. Journal of Gambling Studies, 21, 157-178.

Prochaska, J. O., \& DiClemente, C. C. (1986). The transtheoretical approach: Towards a systematic eclectic framework. In J. C. Norcross (Ed.), Handbook of eclectic psychotherapy 
(pp. 163-200). New York: Brunner/Mazel.

Prochaska, J. O., DiClemente, C. C., \& Norcross, J. C. (1992). In search of how people change. Applications to addictive behaviors. American Psychologist, 47, 1102-1114.

Taber, J., \& McCormick, R. (1987). The pathological gambler in treatment. In T. Galski (Ed.). The handbook of pathological gambling (pp. 137-168). Springfield, IL: Charles C. Thomas.

The Hong Kong Polytechnic University (2002). Report on a study of Hong Kong People's participation in gambling activities. Hong Kong: The Hong Kong Polytechnic University. Retrieved 2nd July, 2010 from http:/ / www.hab.gov.hk/file_manager/en/documents / whats_new/gambling/report-eng.pdf

The University of Hong Kong (2005). Report on a study of Hong Kong people's participation in gambling activities. Hong Kong: The University of Hong Kong. Retrieved $8^{\text {th }}$ July, 2010 from: http://www.hab.gov.hk/file_manager/en/documents/whats_new/gambling/ KeyStat_200514_e.pdf

Toneatto, T., \& Millar, G. (2004). Assessing and treating problem gambling: Empirical status and promising trends, Canadian Journal of Psychiatry, 49, 517-525.

Wong, I. (2010). Hong Kong gamblers'self-help groups: Contributions and challenges. Paper presented at the International Conference on Gaming Industry and Public Welfare 2010, Beijing, China.

Wong, Y. L. R., Leung, Y. K. T., \& Lau, C. W. D. (2009). Behind the allure of gambling: A qualitative exploration of the existential yearnings of Chinese men with problem gambling in Hong Kong. International Gambling Studies, 9, 189-205.

Wood, R. T. A., \& Griffiths, M. D. (2007). A qualitative investigation of problem gambling as an escape-based coping strategy. Psychology and Psychotherapy: Theory, Research and Practice, $80,107-125$. 